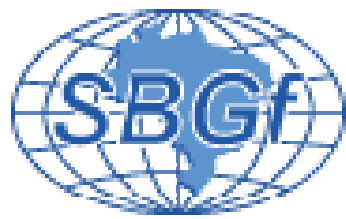

\title{
Condições de fronteira para as equações unidirecionais de amplitude verdadeira
}

Flor Alba Vivas e Reynam Pestana, CPGG - Universidade Federal da Bahia

Copyright 2006, SBGf - Sociedade Brasileira de Geofísica

Este texto foi preparado para a apresentação no II Simpósio de Geofísica da Sociedade Brasileira de Geofísica, Natal, 21-23 de setembro de 2006. Seu conteúdo foi revisado pela Comissão Tecno-científica do II SR-SBGf mas não necessariamente representa a opinião da SBGf ou de seus associados. E proibida a reprodução total ou parcial deste material para propósitos comerciais sem prévia autorização da SBGf.

\section{Resumo}

Os operadores unidirecionais da equação da onda têm se mostrado eficiente no imageamento de estruturas complexas, entretanto as amplitudes das imagens migradas não correspondem às obtidas através de um procedimento de migração/inversão tipo Kirchhoff. A fim de obter amplitude corretas, Zhang et al. (2001) introduziram modificações aos operadores unidirecionais. Em Zhang et al. (2005) também é apresentada uma nova formulação da migração, utilizando esses novos operadores unidirecionais, com condições de imagem e de fronteira modificadas. Neste trabalho, usando alguns exemplos numéricos 2D, mostramos que a modificação da condição de fronteira é um problema crítico na obtenção de amplitudes verdadeiras e que no caso homogêneo, essas condições de fronteira, quando implementadas adequadamente com a condição de imagem modificada, são suficientes para a obtenção de resultados com amplitudes verdadeiras.

\section{Introdução}

Os operadores unidirecionais da onda, obtidos mediante a fatoração da equação completa da onda, para um meio em que a velocidade varia apenas com a profundidade, $v(z)$, permitem decompor o campo de ondas em campos ascendente $(U)$ e descendente (D) e extrapolá-los no domínio freqüência-número de onda $(\omega-k)$. Esta decomposição ainda é válida, com boa aproximação, para meios heterogêneos, $v(x, y, z)$, ou seja, com variações laterais de velocidades suaves, e, ainda assim, obtendo-se imagens migradas de alta qualidade, por levar em conta as múltiplas chegadas, como mostrado em Stoffa et al., (1990), Gazdag (1984) e Claerbout (1985).

Entretanto, a parte dinâmica dos operadores unidirecionais não corresponde com a obtida mediante a solução da equação da completa da onda com aproximação de altas freqüências, como mostrado por Zhang et al. (2001). No intuito de obter operadores unidirecionais que mantenham a fidelidade cinemática e dinâmica da equação completa da onda equivalentes às obtidas por aproximação de altas freqüências, Zhang et al. (2005) apresentaram um esquema de migração com operadores unidirecionais modificados e também novas condições de imagem e de fronteira, porém válidos apenas para dados sísmicos ordenados em tiro comum com um arranjo de receptores cobrindo toda a extensão do levantamento.

Neste trabalho, mostramos que, no caso de um meio homogêneo, somente é necessário modificar as condições de fronteira e a condição de imagem para os campos ascendente e descendente redefinidos e, assim, obter corretamente as amplitudes. Também mostramos que para o caso de refletores horizontais num meio homogêneo a modificação na condição de fronteira corresponde com a formulação proposta por Wapenaar (1990), para conseguir modelar corretamente uma fonte linear de injeção de volume com operadores unidirecionais. No entanto, para refletores inclinados aparece claramente que a condição de fronteira para amplitude verdadeira deve ser aplicada nos dados, se a extrapolação é feita diretamente nos campos $D$ e $U$.

Apresentamos dois exemplos numéricos, o primeiro com refletores horizontais em diferentes profundidades e o segundo com um refletor inclinado, mostrando que os melhores resultados na imagem migrada $e$ na recuperação das amplitudes são obtidos quando a condição de fronteira modificada é aplicada diretamente aos dados.

\section{Migração de tiro comum com amplitude verdadeira}

A migração pré-empilhamento, para dados de tiro comum, é formulada a partir da solução da equação acústica da onda, com um valor na fronteira determinado, onde cada tiro corresponde a um experimento físico independente. Dado o campo de onda acústico $p(x, y, z=0 ; t)$, registrado na superfície, e a equação de onda com fonte impulsiva na posição $\left(x_{s}, y_{s}, z=0\right)$, em $t=0$, encontra-se a solução $P(x, y, z ; \omega)$ de

$\frac{\omega^{2}}{v^{2}}+\frac{\partial^{2} P}{\partial z^{2}}+\Delta P=-\delta\left(x-x_{s}, y=y_{s}, z\right)$,

onde $\Delta=\frac{\partial^{2}}{\partial x^{2}}+\frac{\partial^{2}}{\partial y^{2}}$ e a com a condição de fronteira dada por:

$P(x, y, z=0 ; \omega)=Q\left(x_{r}, y_{r} ; \omega\right)$.

Bleinstein et al. $(1987,2001)$ apresentaram uma equação geral para a migração/inversão Kirchhoff 3D das equações (1) e (2) em meios heterogêneos. No caso em que a velocidade varia com a profundidade, $v(z)$, Zhang 
et al. (2000) conseguiram expressões simplificadas para os pesos na migração Kirchhoff, sendo que para o caso 2D a fórmula de migração pré-empilhamento é dada por:

$$
R(x, z) \approx \iint i \omega \frac{\sqrt{\cos \alpha_{s o} \cos \alpha_{r o}}}{v_{o}} \sqrt{\frac{\psi_{s}}{\psi_{r}}} e^{i \omega\left(\tau_{s}+\tau_{r}\right)} Q\left(x_{r} ; \omega\right) d x_{r} d \omega,
$$

onde $\psi$ é o espalhamento geométrico da fonte linear, no plano $x z, \alpha_{\text {so }}$ e $\alpha_{\text {ro }}$ são os ângulos que o raio forma com a vertical, na superfície na posição do tiro e do receptor, $\alpha_{s}$ e $\alpha_{r}$ são os correspondentes ângulos em subsuperfície e $v_{o}$ a velocidade na superfície (ver Figura 1).



Figura 1 - trajetória do raio num meio $v(z)$.

$\mathrm{Na}$ migração de tiro comum convencional 2D, através das equações unidirecionais, o campo descendente $D$ e ascendente $U$ são extrapolados para baixo mediante as equações:

$$
\begin{aligned}
& \left(\frac{\partial}{\partial z}+i \lambda\right) D=0, \\
& D(x, z=0 ; \omega)=\delta\left(x-x_{s}, z\right),
\end{aligned}
$$

e

$\left(\frac{\partial}{\partial z}-i \lambda\right) U=0$

$U(x, z=0 ; \omega)=Q(x ; \omega)$,

onde $\lambda=\frac{\omega}{v} \sqrt{1+\frac{v^{2}}{\omega^{2}} \frac{\partial^{2}}{\partial x^{2}}}$.

Para produzir a imagem é usada a condição

$$
R(x, z)=\int \frac{U(x, z ; \omega)}{D(x, z ; \omega)} d \omega .
$$

Em meios $v(z)$, Zhang et al. (2001) apresentaram as seguintes expressões assintóticas para os campos de onda descendente e ascendente:

$$
D(x, z ; \omega) \approx \frac{i \omega}{2 \pi} \sqrt{\frac{\cos \alpha_{s}}{\psi_{s}}} e^{-i \omega \tau_{s}},
$$

e

$$
U(x, z ; \omega) \approx \int \frac{i \omega}{2 \pi} \sqrt{\frac{\cos \alpha_{r}}{\psi_{r}}} e^{i \omega \tau_{r}} Q d x_{r} .
$$

Substituindo as equações (7) e (8) na condição de imagem (6), obtemos

$$
R(x, z)=\iint \sqrt{\frac{\cos \alpha_{r}}{\cos \alpha_{s}}} \sqrt{\frac{\psi_{s}}{\psi_{r}}} e^{i \omega\left(\tau_{s}+\tau_{r}\right)} Q d x_{r} d \omega .
$$

Comparando (9) com (3), conclui-se que as equações (46) não produzem uma imagem com amplitude equivalente à obtida mediante a migração/inversão Kirchhoff em meios $v(z)$.

Zhang (1993) demonstrou também que $U$ e $D$ não são componentes de $p$, mas que estão relacionados através de:

$$
\begin{aligned}
& D+U=i \lambda p, \\
& D=\frac{1}{2}\left(i \lambda-\frac{\partial}{\partial z}\right) p, \\
& U=\frac{1}{2}\left(i \lambda+\frac{\partial}{\partial z}\right) p,
\end{aligned}
$$

Decorre assim que as condições de fronteira de (4) e (5), e a condição de imagem (6) devem ser substituídas por

$$
\begin{aligned}
& \left(\frac{\partial}{\partial z}+i \lambda\right) D=\frac{1}{2} \delta\left(x-x_{s}, z\right), \\
& \left(\frac{\partial}{\partial z}-i \lambda\right) U=\frac{1}{2} \delta\left(x-x_{s}, z\right), \\
& \left.(U+D)\right|_{z=0}=\left.i \lambda\right|_{z=o} Q(x ; \omega),
\end{aligned}
$$

e, além disso, com o intuito de se obter as equações homogêneas para $D$ e $U$, fazemos

$$
\begin{aligned}
& \left.D\right|_{z=O}=\frac{1}{2} \delta\left(x-x_{S}\right), \\
& \left.U\right|_{z=O}=\left.i \lambda\right|_{z=O} Q(x ; \omega),
\end{aligned}
$$

com a condição de imagem dada por:

$$
R(x, z) \approx \int \frac{p_{U}(x, z ; \omega)}{p_{D}(x, z ; \omega)} d \omega .
$$

onde,

$$
\begin{aligned}
& p_{D}=(i \lambda)^{-1} D, \\
& p_{U}=(i \lambda)^{-1} U .
\end{aligned}
$$

Substituindo-se as expressões assintóticas (7) e (8) para $D$ e $U$ em (13) e observando que $\lambda=\frac{\omega}{v} \cos \alpha$, obtém-se

$$
\begin{aligned}
& R(x, z)=\iint \frac{\lambda_{s}}{\lambda_{r}} \sqrt{\frac{\cos \alpha_{r}}{\cos \alpha_{s}}} \sqrt{\frac{\psi_{s}}{\psi_{r}}} \\
& e^{i \omega\left(\tau_{s}+\tau_{r}\right)} i \lambda_{r o} Q\left(x_{r} ; \omega\right) d x_{r} d \omega,
\end{aligned}
$$

ou

$$
\begin{aligned}
& R(x, z)=\iint \sqrt{\frac{\cos \alpha_{s}}{\cos \alpha_{r}}} \sqrt{\frac{\psi_{s}}{\psi_{r}}} \\
& e^{i \omega\left(\tau_{s}+\tau_{r}\right)} i \frac{\omega}{v_{o}} \cos \alpha_{r o} Q\left(x_{r} ; \omega\right) d x_{r} d \omega .
\end{aligned}
$$


Para um meio homogêneo temos que $\alpha_{s}=\alpha_{\text {so }}$ e $\alpha_{r}=\alpha_{\text {ro }}$, nestas condições a equação (15) se reduz à equação (3).

O algoritmo para migração "Phase-shift" com amplitude verdadeira, num meio com velocidade constante, consiste então das seguintes etapas:

1. Modificação da condição de fronteira dos dados registrados nos receptores.

2. Extrapolação dos campos $D$ e $U$ com a migração "Phase-shift" convencional.

3. Obtenção dos campos $p_{D}$ e $p_{U}$ em cada nível em profundidade.

4. Condição de imagem (13) em cada nível em profundidade.

Nota-se que a condição de fronteira em (12) é equivalente com à proposta por Wapenaar (1990) para modelar uma fonte linear de injeção de volume com as equações unidirecionais, se aplicada somente aos campos $p_{U}$ e $p_{D}$ e a extrapolação é feita diretamente nesses campos, como mostrado por Zhang et al.(2005). Se a condição de fronteira de Wapenaar (1990) é aplicada aos campos $D$ e $U$, ou seja:

$$
\begin{aligned}
& \left.D\right|_{z=o}=\frac{1}{2 i \lambda} \delta\left(x-x_{s}\right), \\
& \left.U\right|_{z=o}=Q(x ; \omega),
\end{aligned}
$$

embora consiga melhorar o padrão de diretividade da fonte, porém não proporciona uma equivalência da equação (13) com a (3).

\section{Experimentos numéricos}

Para ilustrar como as condições de fronteira (12) e (16) afetam a imagem e a amplitude dos refletores migrados em um meio homogêneo foi gerado um modelo com quatro refletores horizontais nas profundidades $z=1000$ m, $2000 \mathrm{~m}, 3000 \mathrm{~m}$ e $4000 \mathrm{~m}$. Velocidade do meio de $v=2000 \mathrm{~m} / \mathrm{s}$. Os quatro refletores modelam variações de densidade e por isso o coeficiente de reflexão recuperado não deve depender do ângulo de reflexão $\theta$.

Os testes foram feitos com um único tiro na posição zero e receptores em afastamentos de $-6 \mathrm{~km}$ a $6 \mathrm{~km}$, com espaçamento uniforme de $20 \mathrm{~m}$, utilizando-se como fonte um pulso "Ricker" de fase zero e freqüência $25 \mathrm{~Hz}$.

Nas Figuras 2a - 2c mostramos as imagens migradas com "Phase-shift" convencional, "Phase-shift" modificando a condição de fronteira nos dados e a condição de imagem (13) e "Phase-shift" modificando a condição de fronteira na fonte e a condição de imagem, eq. (13), respectivamente. Observa-se que embora nos três casos a parte cinemática permaneça correta, a informação de amplitude varia grandemente em todos os casos testados.
Nas Figuras $3 a-3 c$ observamos as amplitudes recuperadas nos refletores com os três métodos. Observa-se uma boa correção das amplitudes com o ângulo no caso em que a condição de fronteira é modificada nos receptores, Figura $3 b$. As amplitudes recuperadas para a modificação da condição de fronteira na fonte são bem comportadas no primeiro refletor. Observamos que isto é produzido pelo fato de ser os refletores horizontais e $\theta=\alpha_{s}=\alpha_{\text {so }}=\alpha_{r}=\alpha_{\text {ro }}$.

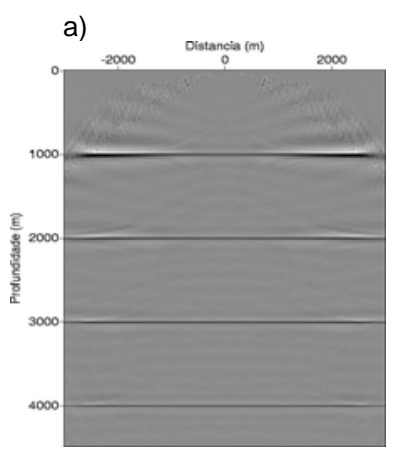

b)
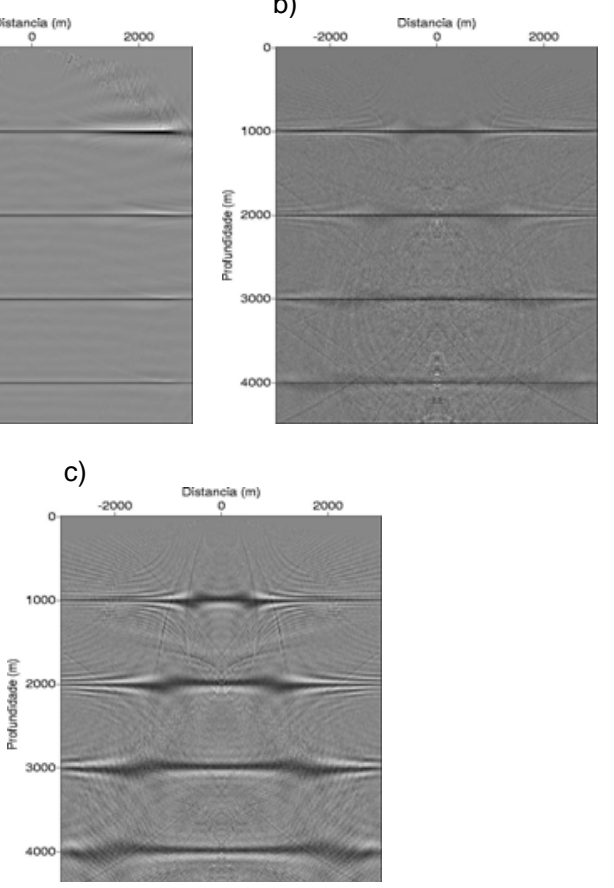

Figura 2 - (a) Imagem do tiro migrado em profundidade com Phase-shift convencional. (b) Modificação da condição de fronteira dos dados. (c) Modificação da condição de fronteira da fonte.

No segundo exemplo os dados foram gerados com a mesma fonte, geometria de aquisição, e mesma velocidade do meio. O modelo tem um refletor inclinado com um mergulho de aproximadamente $18^{\circ}$, portanto, com $\alpha_{s}=\alpha_{\text {so }} \neq \alpha_{r}=\alpha_{r o}$. Os resultados da migração, Figuras $4 \mathrm{a}-4 \mathrm{c}$, mudam significativamente em relação à modificação da condição de fronteira. Neste modelo somente a modificação da condição de fronteira aplicada aos dados produz o resultado correto em amplitude (Figura 4b).

\section{Discussão e conclusões}

Mostramos neste trabalho que em meios homogêneos, a modificação da condição de fronteira e também da condição de imagem são suficientes para recuperar corretamente as amplitudes, mesmo com o método de migração "Phase-shift" convencional, se a condição de fronteira modificada for aplicada de forma correta nos receptores quando extrapolamos os campos $U$ e $D$. Se a extrapolação é feita diretamente aos campos $p_{U}$ e $p_{D}$, usando-se os operadores unidirecionais derivados por Zhang et al. (2005), esta condição de fronteira equivale a modificação proposta por Wapenaar (1990) para a 
modelagem correta de uma fonte linear de injeção de volume com operadores unidirecionais.

\section{Agradecimentos}

Os autores agradecem ao suporte do LAGEPCPGG/UFBA para o desenvolvimento deste trabalho e F. Vivas agradece à Universidade de Pamplona - Colômbia pelo suporte financeiro.

a)

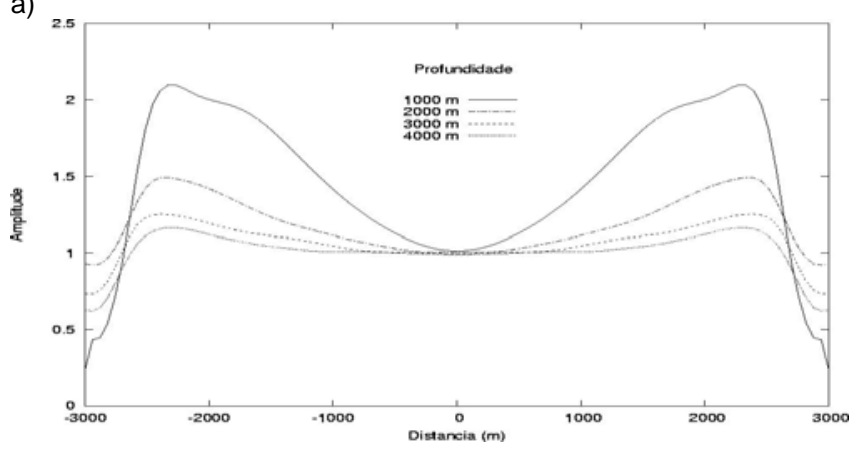

b)



c)



Figura 3 - (a) Picos de amplitudes com a migração Phase-shift convencional. (b) modificação da condição de fronteira nos dados. (c) modificação da condição de fronteira na fonte.

\section{Referências}

Bleistein, N., 1987. On the imaging of reflectors on the earth. Geophysics, vol. 52, NO. 7: 931-942.

Bleistein, N., J. K. Cohen, and J. W. Stockwell, 2001. Mathematics of multidimensional seismic inversion: Springer Publishing Co.

Claerbout, J. F., 1985. Imaging the earth's interior: Blackwell Scientific Publications, Inc.
Gazdag, J., P., Sguazzero, 1984. Migration of seismic data by phase shift plus interpolation. Geophysics, vol. 49, NO. 2: 124 131.

Stoffa, P., J. T. Fokkema, R. M. de Luna Freire, and W. P. Kessinger, 1990. Split-step Fourier migration. Geophysics, vol. 55, NO. 4: $410-421$.

Wapenaar, C., 1990. Representation of seismic sources in the one-way wave equations. Geophysics, vol. 55, NO. 6: 786-789

Zhang, Y., J. Sun, S. Gray, C. Notfors, 2001. Towards accurate amplitudes for one-way wavefield extrapolation of 3-D common shot records, presented at workshop W-7, $71^{\text {st }}$ Annual International Meeting, SEG.

Zhang, Y., G. Zhang, and N. Bleistein, 2005. Theory of trueamplitude one-way wave equations and true- amplitude common-shot migration. Geophysics, vol. 70, NO. 4:E1-E10.

a)

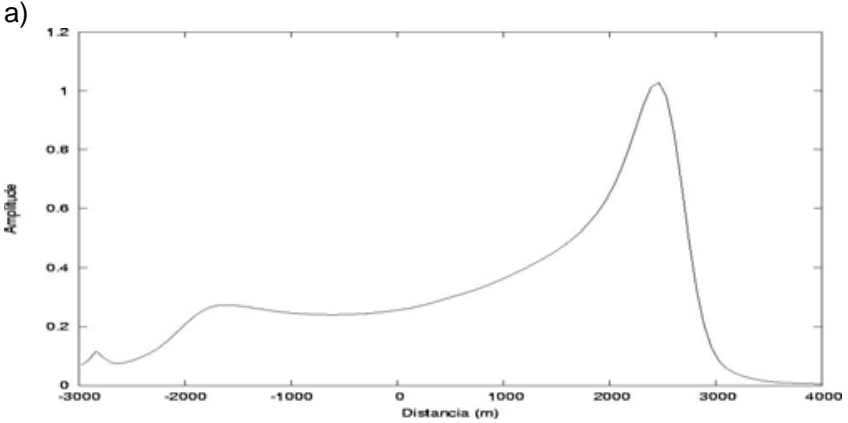

b)

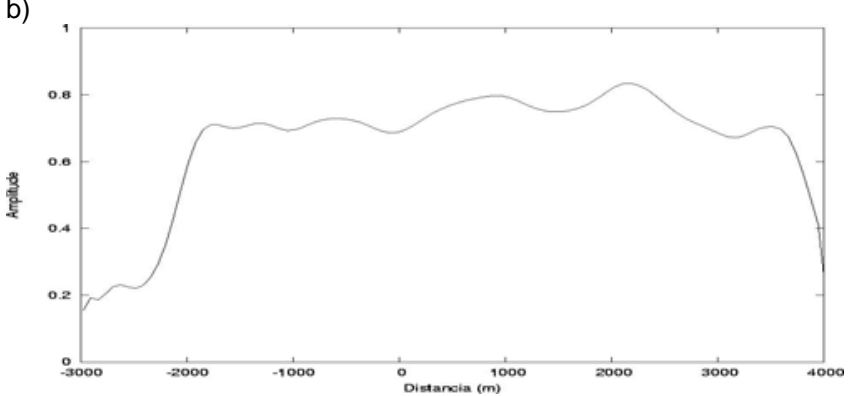

c)

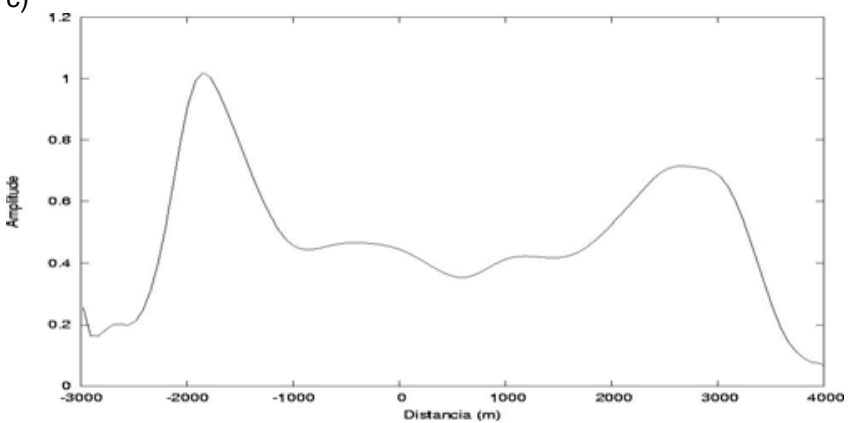

Figura 4 - (a) Picos de amplitudes na migração phase shift convencional. (b) modificação da condição de fronteira no dado. (c) modificação da condição de fronteira na fonte. 